\title{
Spontaneous atrial fibrillation after long-term transesophageal atrial burst pacing in rats. Technical and procedural approach to a new in vivo atrial fibrillation model
}

\author{
Alina Scridon ${ }^{1,2 *, \#}$, Marcel Perian ${ }^{1, \#}$, Teodor Grigoraș ${ }^{1}$, Vasile Bogdan Halaţiu ${ }^{1}$, \\ Adriana Vântu ${ }^{1}$, Alkora Ioana Balan ${ }^{1}$, Ionela Alexandra Cosma ${ }^{1}$, Asmaa Carla \\ Barmou$^{1}$, Bogdan Andrei Finascu ${ }^{1}$, Diana Lavinia Moldovan ${ }^{1}$, Dan Alexandru \\ Cozac $^{1}$, Răzvan Constantin Șerban ${ }^{1,3}$

\section{Physiology Department, University of Medicine and Pharmacy of Tirgu Mures, Romania \\ 2. Center for Advanced Medical and Pharmaceutical Research, Tirgu Mures, Romania} \\ 3. Emergency Institute for Cardiovascular Diseases and Transplantation Tirgu Mures, Romania
}

\section{Background}

In vivo animal models that replicate human conditions are invaluable scientific tools. Models that mimic human diabetes, dyslipidemia, or myocardial infarction (1-3), have provided the background for our understanding regarding the pathophysiology of such conditions and have often represented the starting point for new therapeutic approaches.

Atrial fibrillation (AF) is the most common sustained cardiac arrhythmia, affecting $2 \%$ of the general population, and up to $17 \%$ of individuals above the age of 80 (4). Several in vivo AF models have been developed and characterized over the past decades. Due to lower costs, larger availability, and shorter study duration, small animals are usually preferred when it comes to experimental disease models. However, the long belief that reentrant arrhythmias, including AF, require a minimum tissue mass, restricted until recently such models to large animals $(5,6)$. Accumulating data demonstrate however that $\mathrm{AF}$ can be induced in small hearts (7). Moreover, in the presence of substrate-modifying factors such as aging and hypertension, rat hearts develop spontaneous AF (8). Although it replicates well the human condition, AF occurrence in this latter model requires long-term (>12 months) follow-up (8).

Alternatively, AF can be induced in small rodents using atrial burst pacing $(9,10)$. However, in the AF models developed to date, the arrhythmia was typically induced by atrial pacing during perfusion with cholinergic agonists or asphyxia $(9,10)$, which have the potential for confounding interactions. Furthermore, all previous

\footnotetext{
* Corresponding author: Alina Scridon, University of Medicine and Pharmacy of Tirgu Mures, Tirgu Mures, Romania. E-mail address: alinascridon@gmail.com

\# The first two authors contributed equally to the manuscript.
} 
studies have focused on AF inducibility after single-burst pacing protocols, and none has evaluated the impact of long-term atrial pacing on arrhythmia inducibility. Also, no study has evaluated to date the occurrence of spontaneous AF following atrial burst pacing in small animals. In contrast to spontaneous AF models, acute AF models do not allow evaluation of AF-related electrical, structural, autonomic, or molecular remodeling, and can only be used to test the acute effects of antiarrhythmic strategies, but not the effects of such strategies on atrial remodeling, or on the risk of ventricular pro-arrhythmia. Therefore, there is an obvious need to develop simple, reproducible, and more clinically-relevant in vivo models of AF in small animals.

In this paper, we describe a new model of spontaneous AF after long-term transesophageal atrial burst pacing in rats, and we provide technical and procedural insights into this model.

\section{Technical requirements}

\section{Development of a microcontroller-based cardiac pacemaker for induction of atrial fibrillation}

Few commercially available pacemakers are able to deliver the high-frequency stimuli $(\geq 4,000 \mathrm{ppm})$ required for $\mathrm{AF}$ induction. Therefore, a digital microcontroller-based external pacemaker was designed using the PIC16F873 MCU (Microchip Technology, Chandler, AZ). The microcontroller has $7 \mathrm{~KB}$ program memory, 128 bytes electrically erasable programmable read-only memory to store predefined data when the device is powered off, and is capable to execute 5 million instructions per second. It disposes of two analog inputs with 8 bit integrated analog-to-digital converters.

The first analog channel (AN0/PIN2) was used to continuously monitor the amplitude of the delivered stimuli and the second (AN1/PIN3) to monitor the battery status during stimulation.
Calibration of stimulation amplitude and battery voltage was done via VR2 and VR4 variable resistors. The pacemaker is programmable using a classical 4-steps rotary encoder. The pushswitch of the encoder stores the stimulating protocol into the non-volatile memory for later use. A 16x2 alphanumeric liquid-crystal display allows the user to monitor impulse width and amplitude, stimulation period and frequency. Three digital outputs of the microcontroller were used for audio (RC5/PIN16) and video (RC4/PIN15) monitoring of the stimuli, and for delivering the stimuli (RC7/PIN18). The schematic design (Figure 1) was made in Eagle CAD and the software was developed in Proton BASIC Pro.

Stimulation can be performed at a maximum frequency of $8,000 \mathrm{ppm}$, with stimulus width $\geq 1$ ms. Ten memory locations are available for predefined settings for each stimulated animal. The device is operated from lithium-ion rechargeable batteries and has low power consumption ( $\approx 600 \mathrm{~mA}$, depending on stimulation frequency, threshold, and impulse width).

\section{Technical approach to surface ECG re- cording in small rodents}

In human ECG recordings, impedances vary between $1 \mathrm{~K} \Omega$ and $50 \mathrm{~K} \Omega$ depending on electrode area, metal-skin interface product, electrode potential (Ag- $\mathrm{AgCl}$ has lower electrode potential), skin thickness and structure. In rodents, the relatively small contact area on the limbs, increased skin density, and lack of local humidity lead to considerably higher impedances (100-500 K $\Omega$ ), and, consequently, to ECG signal loss and increased environmental electrical noise. Such drawbacks are even more important when analysis of low-amplitude atrial electrical signals is desired. In rodents, AF assessment using surface ECG recordings requires adequate amplification. Accordingly, an ECG amplification system was developed to allow AF assessment during atrial pacing protocols. 


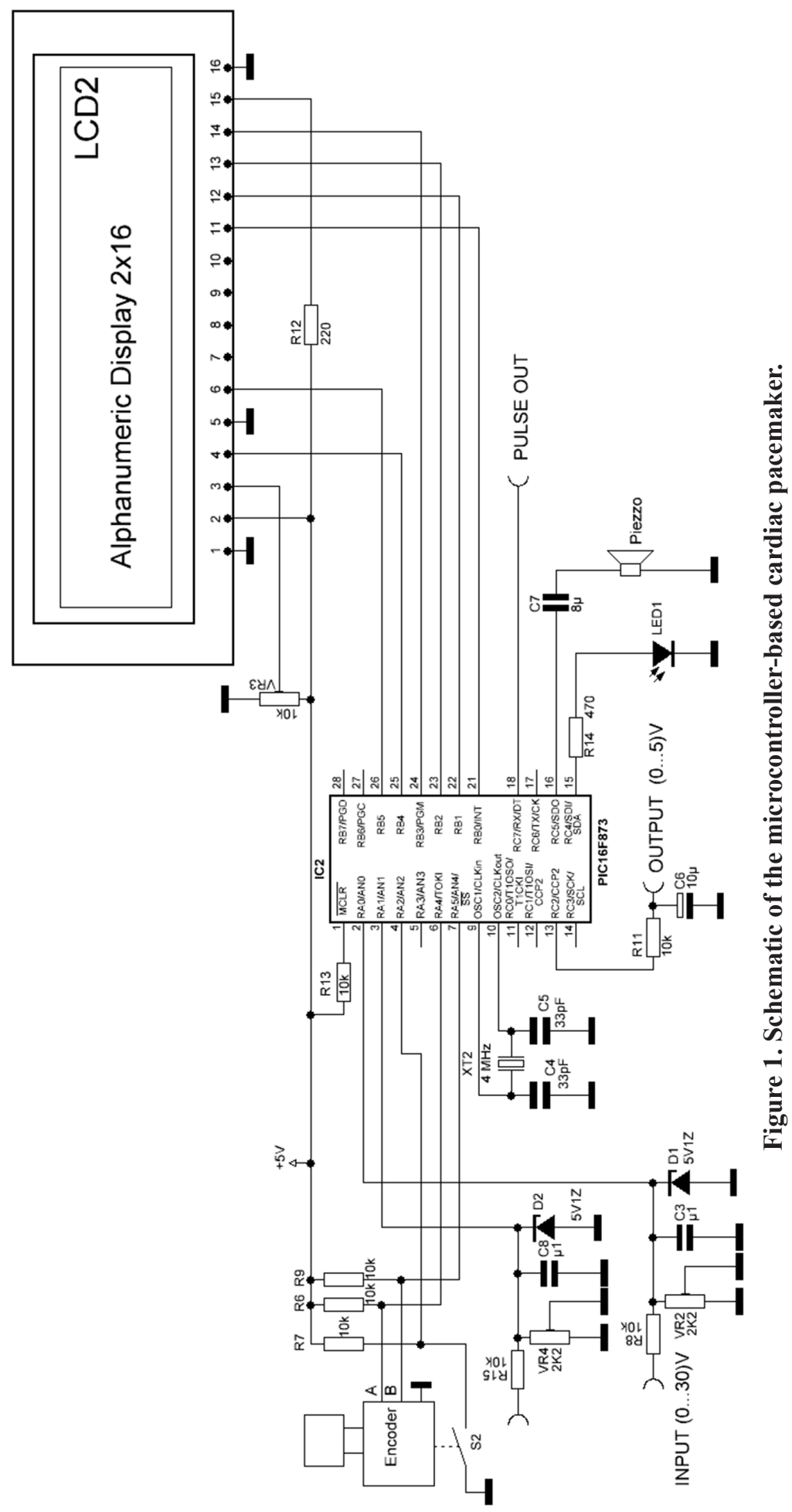

The AD621 low drift instrumentation amplifier (Analog Devices, Norwood, MA) was used as main amplifier chip due to its very high common-mode rejection ratio $(110 \mathrm{~dB})$, which allows efficient suppression of parasitic electric signals having the same phase on both input leads. The AD621 amplifier also has very low internal noise $(<9$ $\mathrm{nV} / \sqrt{\mathrm{Hz}}$ ) and draws only 1.3 $\mathrm{mA}$, allowing it to operate continuously for several days from batteries. The gain, which is selectable using a simple resistor, can vary between $10 x$ and $100 x$. Continuous gain variation is also possible using variable resistor between pins 1 and 8 of the chip.

Given that the input impedance imbalance between electrodes should not exceed $1 \mathrm{~K} \Omega$ (11), in rodents, the AD621 amplifier cannot be used alone. To preserve the good performances of the instrumentation amplifier, a high-impedance buffer was designed using the LMC6484 quadruple operational amplifier (Texas Instruments, Dallas, TX). The first two operational amplifiers were used as buffers, offering an input impedance in the range of $10 \mathrm{~T} \Omega$, for an output impedance of $2 \mathrm{~K} \Omega$. When compared to the input impedances of the buffer, imbalances of tens or hundreds of $\mathrm{K} \Omega$ are negligible, allowing the instrumentation amplifier to operate within optimal parameters. Isoelectric line 


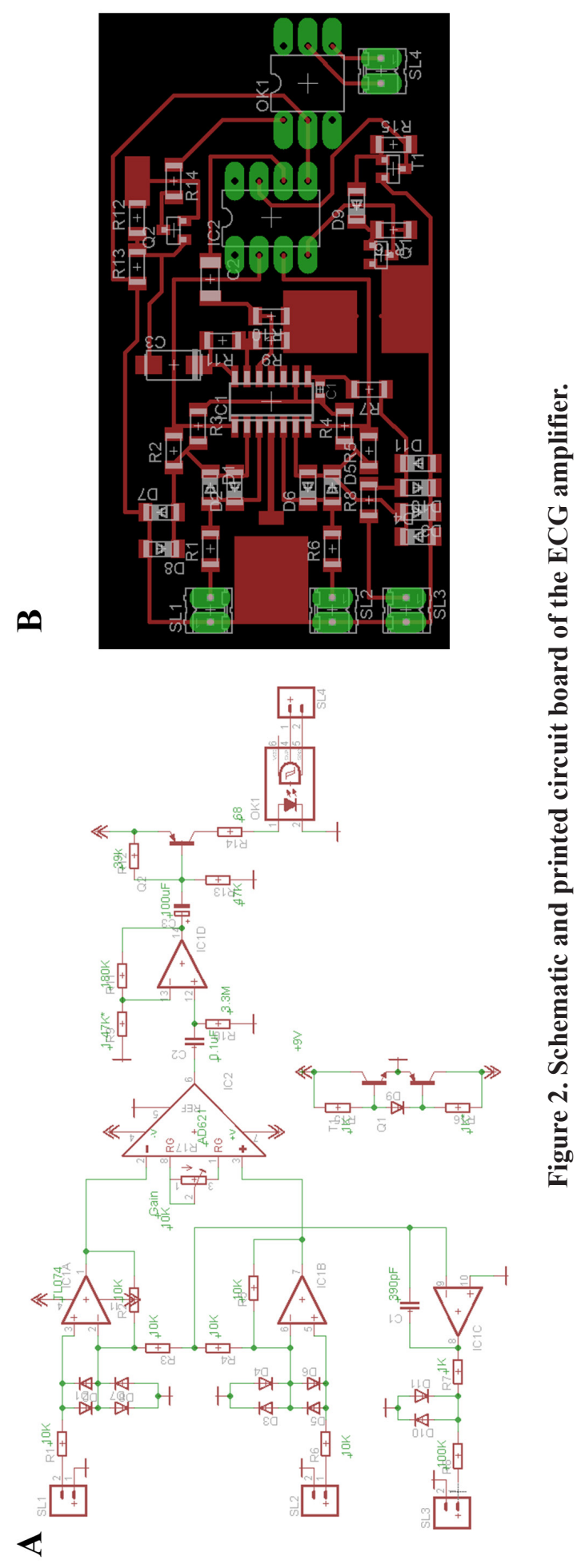

stabilization is obtained automatically, using the third operational amplifier, which drives the third limb of the animal at a potential equal and opposite to the medium potential of the active limbs. The amplifier schematic (Figure 2A) and printed circuit board (Figure 2B) were designed in Eagle CAD. The amplifier was insulated electrically using the OK1 optocoupler (LEG Industrie-Elektronik GmbH, Viersen, Germany), ensuring $3 \mathrm{KV}$ separation between the animal and the acquisition setup. The amplifier ground was generated using two transistors, allowing the setup to be operated using a single power supply.

\section{Transesophageal atrial burst pacing}

The protocol was developed and validated using nine adult male Wistar rats (250-350 g) obtained from the Experimental Animal Center of the University of Medicine and Pharmacy of Tîrgu Mureș, Romania. All animals were housed individually in a climate-controlled room (21$24^{\circ} \mathrm{C}$ ), with a $12 / 12-\mathrm{h}$ light/dark cycle, and had free access to food and water. All rats were implanted at the beginning of the study with radiotelemetry ECG devices (TA11 CA-F40; Data Sciences International, St. Paul, MN), as described previously $(8,12)$.

Rats were anesthetized with ketamine/medetomidine (75.0/0.5 mg/kg, i.p.). A 6-French quadripolar catheter connected to the stimulator was inserted into the esophagus under continuous surface ECG monitoring. The stimulation catheter was positioned to ensure constant capture of the atria (i.e., each stimulus is followed by a narrow QRS complex). The correct positioning of the catheter can be confirmed during the protocol, by the presence of a short pause (i.e., sinus node recovery time) after each stimulation cycle (Figure 3A).

After setting the position of the catheter, the atria were stimulated at a rate of 400 ppm (Figure $3 \mathrm{~A}$ ) and the diastolic threshold was deter- 

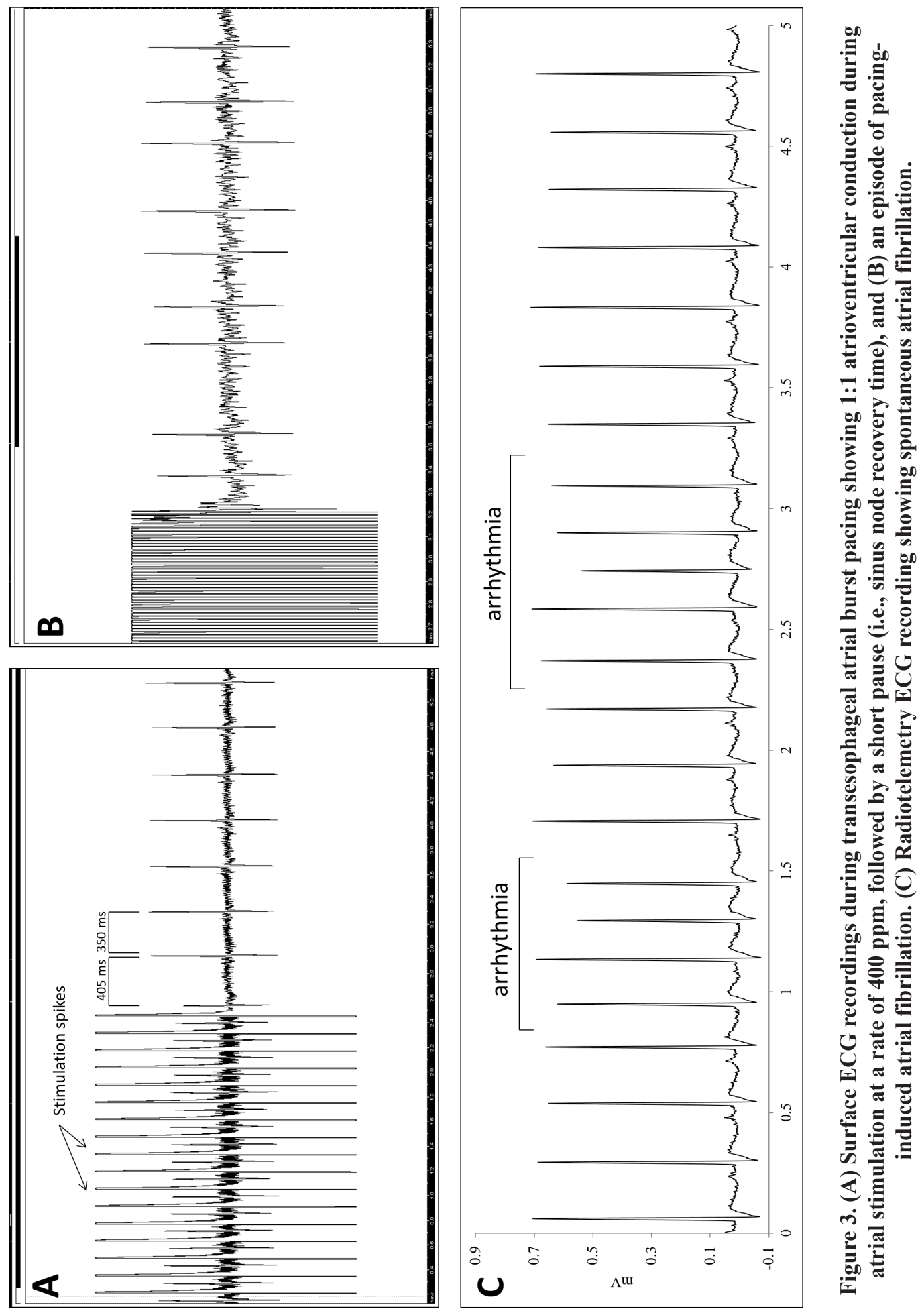
mined as the lowest voltage ensuring capture of the atria $(\approx 10-20 \mathrm{~V})$. The pacing pulse was rectangular in shape and pulse width was $6 \mathrm{~ms}$. The rats were then stimulated at a rate of $4,000 \mathrm{ppm}$, at a voltage $\approx 3 \mathrm{~V}$ above the diastolic threshold. Burst pacing was applied for 15 consecutive cycles, $20 \mathrm{~s}$ each, with 5-min intervals between cycles. The protocol was repeated five days/week, for two consecutive weeks. If pacing-induced AF lasted longer than $10 \mathrm{~min}$, the stimulation protocol was abandoned that day. At the end of the protocol, anesthesia was reversed with $1 \mathrm{mg} /$ $\mathrm{kg}$ atipamezole.

All experiments complied with the International Council for Laboratory Animal Science guidelines (Directive 2010/63/EU) and were approved by the local Ethics Committee.

Given the close proximity of the vagus nerve to the esophagus, the major concern during transesophageal pacing is concomitant vagal stimulation, occasionally followed by severe bradycardia and even asystole. In such cases, atropine administration, associated with external cardiac massage, if required, was usually effective in restoring normal sinus rhythm. In such cases, the stimulation protocol was aborted for that day, as recurrent episodes of vagal hyperactivity often occur in this setting.

\section{Atrial fibrillation inducibility}

Electrocardiographic tracings recorded during the stimulation procedures can be used to assess arrhythmia inducibility and duration. Arrhythmia inducibility (i.e., number of stimulation cycles followed by AF/total number of stimulation cycles) can be assessed separately for each day of stimulation, or can be expressed as overall AF inducibility (i.e., number of stimulation cycles followed by AF/total number of stimulation cycles applied during the entire protocol). Given that during pacing waves are difficult, if not impossible, to recognize, AF duration is usually measured from the end of burst pacing to the first $\mathrm{P}$ wave detected after the irregular supraventricular rhythm. In accordance with previous studies (8), AF was defined in this study as rapid, irregular, supraventricular rhythm, with no visible $\mathrm{P}$ waves and irregular R-R intervals, lasting at least three beats. In other studies, a duration of at least $2 \mathrm{~s}$ was required to define $\mathrm{AF}$ (10).

In the present cohort, AF was induced in the first day of stimulation in six of the nine studied rats $(66.7 \%)$. Out of the 98 stimulation cycles applied in the first day, 18 (18.4\%) efficiently induced AF (i.e., first day inducibility). In three of the nine rats, the protocol was stopped prematurely because pacing-induced AF lasted longer than $10 \mathrm{~min}$. Atrial fibrillation was induced in all animals (Figure 3B) during the first week of stimulation. In total, 633 stimulation cycles were applied throughout the protocol, and 113 of them $(17.9 \%)$ were followed by AF (overall inducibility). Vagal reactions requiring atropine administration occurred in two of the nine rats.

\section{Spontaneous atrial fibrillation occurrence after long-term atrial burst pacing}

After the two weeks of pacing, 72-h continuous ECG monitoring performed in freely moving rats revealed spontaneous AF occurrence (Figure 3C) in all studied rats (median $19.5 \mathrm{ep}-$ isodes [IQR 10.5-24.0]; median duration $1.14 \mathrm{~s}$ [IQR 0.96-1.22 s]).

\section{Perspectives}

These data show that spontaneous AF occurs in healthy rats after long-term transesophageal atrial burst pacing, in the absence of any adjuvants that may act as clinically non-relevant confounding factors, and illustrate for the first time the 'AF begets AF' concept (5) in rats.

Many therapeutic approaches provided extremely promising results in animal models, but, when applied in clinical settings, they were less 
convincing. Experimental models that do not mimic faithfully the human condition or use of surrogate endpoints (e.g., AF inducibility instead of spontaneous AF occurrence) may be at least partly responsible for these discrepancies. The model described in this paper can be used to assess not only AF-related remodeling, but also the effects of new antiarrhythmic strategies on spontaneous AF occurrence, in the absence of any factor that may interfere with the tested strategy. The protocol can easily be applied in other clinically-relevant models (e.g. models of diseases known to be associated with increased risk of AF, such as aging and hypertension, heart failure, or obesity $[8,13-15])$, or in animals treated with drugs commonly used in AF patients.

\section{Acknowledgements}

This work was supported by a grant of the Romanian National Authority for Scientific Research and Innovation, CNCS-UEFISCDI (PNII-RU-TE-2014-4-1544).

\section{Conflict of interest}

None to declare.

\section{References}

1. Scridon A, Perian M, Marginean A, Fisca C, Vantu A, Ghertescu D, et al. Wistar rats with long-term streptozotocin-induced type 1 diabetes mellitus replicate the most relevant clinical, biochemical, and hematologic features of human diabetes. Rev Romana Med Lab. 2015;23(3):263-84.

2. Zheng D, Liang Q, Zeng FF, Mai Z, Cai A, Qiu R, et al. Atorvastatin protects endothelium by decreasing asymmetric dimethylarginine in dyslipidemia rats. Lipids Health Dis. 2015;14:41.

3. Wu Y, Yin X, Wijaya C, Huang MH, McConnell BK. Acute myocardial infarction in rats. J Vis Exp. 2011;48:2464.
4. Zoni-Berisso M, Lercari F, Carazza T, Domenicucci S. Epidemiology of atrial fibrillation: European perspective. Clin Epidemiol. 2014;6:213-20.

5. Wijffels MC, Kirchhof CJ, Dorland R, Allessie MA. Atrial fibrillation begets atrial fibrillation. A study in awake chronically instrumented goats. Circulation. 1995;92(7):1954-68.

6. Sharifov OF, Fedorov VV, Beloshapko GG, Glukhov AV, Yushmanova AV, Rosenshtraukh LV. Roles of adrenergic and cholinergic stimulation in spontaneous atrial fibrillation in dogs. J Am Coll Cardiol. 2004;43(3):483-90.

7. Schrickel JW, Bielik H, Yang A, Schimpf R, Shlevkov $\mathrm{N}$, Burkhardt D, et al. Induction of atrial fibrillation in mice by rapid transesophageal atrial pacing. Basic Res Cardiol. 2002;97(6):452-60.

8. Scridon A, Gallet C, Arisha MM, Oréa V, Chapuis B, Li N, et al. Unprovoked atrial tachyarrhythmias in aging spontaneously hypertensive rats: The role of the autonomic nervous system. Am J Physiol Heart Circ Physiol. 2012;303(3):H386-92.

9. Wakimoto H, Maguire CT, Kovoor P, Hammer PE, Gehrmann J, Triedman JK, et al. Induction of atrial tachycardia and fibrillation in the mouse heart. Cardiovasc Res. 2001;50(3):463-73.

10. Haugan K, Lam HR, Knudsen CB, Petersen JS. Atrial fibrillation in rats induced by rapid transesophageal atrial pacing during brief episodes of asphyxia: a new in vivo model. J Cardiovasc Pharmacol. 2004;44(1):125-35.

11. http://www.analog.com/media/en/technical-documentation/data-sheets/AD621.pdf. Accessed on November $7^{\text {th }}, 2017$.

12. Scridon A, Fouilloux-Meugnier E, Loizon E, Rome S, Julien C, Barrès $\mathrm{C}$, et al. Long-standing arterial hypertension is associated with Pitx2 down-regulation in a rat model of spontaneous atrial tachyarrhythmias. Europace. $2015 ; 17(1): 160-5$.

13. Scridon A, Tabib A, Barrès C, Julien C, Chevalier P. Left atrial endocardial fibrosis and intra-atrial thrombosis - landmarks of left atrial remodeling in rats with spontaneous atrial tachyarrhythmias. Rom J Morphol Embryol. 2013;54(2):405-11.

14. van Deursen VM, Urso R, Laroche C, Damman K, Dahlström U, Tavazzi L, et al. Co-morbidities in patients with heart failure: an analysis of the European Heart Failure Pilot Survey. Eur J Heart Fail. 2014;16(1):103-11.

15. Scridon A, Dobreanu D, Chevalier P, Șerban RC. Inflammation, a link between obesity and atrial fibrillation. Inflamm Res. 2015;64(6):383-93. 